\title{
A Study on Impacts of Distributed Generation Voltage in Distribution Network System
}

\author{
Wei Song \\ Institute of electrical and electronic engineering \\ North China Electric Power University \\ Baoding, China \\ e-mail: yigenage@126.com
}

\author{
Xiaolong Liu
}

Institute of electrical and electronic engineering

North China Electric Power University

Baoding, China

e-mail: 970466312@qqu.com

\author{
Xinghua Zhou \\ Power grid planning products division \\ Beijing Join Bright Digital Power Technology Limited \\ Company \\ Beijing, China \\ e-mail: 1014433580@qq.com
}

\author{
Hongting Zhou \\ Institute of electrical and electronic engineering \\ North China Electric Power University \\ Baoding, China \\ e-mail: zhouhongting@126.com
}

\begin{abstract}
Distributed generator is often associated with the existing distribution network system, the study of the impact of the distributed generation on original distribution network system is very important for the development of the smart grid. The study on the voltage can contribute to the safety, reliability and efficiency of the distribution network system. As the distributed generations connected to the grid, the calculation of power flow will change. Joining the distribution generation into the distribution network system safely and reliably is significant and this also related to the value of the distributed generation. The impacts of voltage in the distributed system will directly affect quality of power supply and system stability as the injection of the distributed generations. When decided locations of the distributed generations, not only the surrounding energy, transportation, geographical and environmental factors, but also the rationality of the distributed generator location shall be considered. Thus the study of the impact of voltage becomes more and more vital after the injection of distributed generation.
\end{abstract}

Keywords-distributed network; smart grid; voltage; distributed generator; power output

\section{INTRODUCTION}

With the development of traditional energy sources and some drawbacks of the traditional generations, the distributed generation[1] has attracted the worldwide attention for its environmental protection, efficiency and flexibility. As the distribution generation becomes more and more important, it is necessary to study the impacts in the distributed network[2] system caused by the distribution generation. Otherwise the penetration of the distributed generation also causes many problems, among which the impact of voltage is outstanding. Much more attention shall be paid to the change of voltage in the distributed network system. This paper uses hierarchical forward and backward substitution method to study the impact of distributed generation to the voltage of distribution network when the distributed generation size, location, operating mode [3]are different. In this paper IEEE33 bus system is used to testify the result. According to the size and location and power factor of distributed generation, the effects of distributed generation on distribution network voltage is discussed and draw a conclusion.

\section{IMPACTS ON VOLTAGE OF THE DISTRIBUTION NETWORK}

\section{A. The mechanism in the grid voltage changes}

Distribution generator refers to the power of tens of $\mathrm{kW}$ to tens of MW and it often distributed in the vicinity of clean and environmental friendly power generation load. The facilities in the distribution network system can be economically, reliably used.Compared to the traditional centralized power generation, distributed generation has relatively small scale and more close to the users. Generally distribution generators can directly transmit electricity to the nearby users. When distribution generation connected to the grid, the grid will be effected in many aspects such as power quality, system protection and the schedule of the distribution network system.

As the distributed generations connected to the grid, the calculation of power flow will change. Its necessary to take different node types into consideration. Combustion engines and gas turbines and other traditional distributed power generally use the mode of synchronous generator. All synchronous generators and distributed generators connected to the grid by the voltage inverter can be processed into PV node. Photovoltaic system, some wind turbines, micro gas turbines and fuel cells are often connected to the grid by inverter. In this paper we chose the asynchronous wind turbines as the distribution generator to study the change of the voltage in the distribution network system. It can be simplified as a PQ node. Thus the active and reactive power of the wind 
turbines are constant. The wind turbine[4,5] runs at the rated output active power and reactive power and voltage runs in what mode should be analyzed according to the specific circumstance. We chose the (1) to calculate the reactive power of the distributed generator.

$$
Q=\frac{(-1)^{n} P \sqrt{1-(\cos \varphi)^{2}}}{\cos \varphi}
$$

Where $P$ is active power of distributed generator, $Q$ is reactive power, $\cos \varphi$ is power factor. When $n$ is a even number, it is a time lag system. On the contrary, it represents a pull ahead system.

\section{B. Impact of distributed power supply to the system voltage}

The assessment to the voltage in the distribution network includes two aspects.

The distributed power fluctuation and its own stop can affect the distribution network and the users in the system. For the renewable generator, the generation capacity often unstable and frequent starts and stops often occur in some cases. In this case, the distribution network and the users can be affected seriously.

The access of distributed generator adds the shortcircuit capacity. And then the system voltage in tensity, inhibition and weakening the voltage fluctuation problems are strengthened. When a fault occurs, the voltage fluctuation and other problems can be better suppressed compared to the traditional distribution network.

As the largest power surges occur at the access point, using the distribution generator access point as the voltage evaluation point. And the changed voltage value can be expressed by (2).

$$
\begin{aligned}
\Delta U & =\left(R_{S}+j X_{S}\right) \times\left(\Delta_{p}+j \Delta_{q}\right) \\
& =\left|Z_{S}\right|(\cos \phi+j \sin \phi) \times|\Delta|(\cos \theta+j \sin \theta) \\
& =\frac{U 2}{S_{k}} \frac{\Delta S_{n}}{U}[(\cos \phi \cos \theta-\sin \phi \sin \theta)+j(\sin \phi \cos \theta+\cos \phi \sin \theta)]
\end{aligned}
$$

Where $\Delta S_{n}$ is the power injected by the distributed generator, $S_{k}$ is the short-circuit capacity in the access point, $Z_{S}=\left(R_{S}+j X_{S}\right)$ is the equivalent impedance, $\Delta I$ is the short-circuit current amount of change, $\theta$ is the power factor angle, $\phi$ is the grid impedance angle, $U$ is the access point voltage.

According to (2), the voltage shock and fluctuation that caused by the distributed power related to three factors. These includes the injected power change, the short-circuit capacity that incorporated into the network and the distributed power factor.

\section{SimULATION ANALYSIS}

\section{A. Impact on voltage in different capacities}

To analyze the impacts of different DG capacities[6], the number and position of DG should be fixed first. DG is connected as an auxiliary power distribution network support rather than the main power distribution network, so the quantity of the DG is limited.If a distribution network system has too many DG, the relay protection system in the distribution network will be effected. Therefore the position and number of the DG should be considered according to the local natural conditions and security and other factors. In this paper, referring to multiple documents, we select two DGs to injecting the distribution network. Chosen the minimize power loss, limited voltage and active power and reactive power balance as the constraints. According to the genetic algorithm, we calculate the reasonable position and capacities of DGs, based on the conditions the effects on voltage can be studied.

Change the DGs power output ratio to $20 \%, 40 \%, 60 \%$, $80 \%$ respectively. And take the lagging power factor at 0.85 . Table 1 represents the DGs output ratio and capacities, and the simulation result is shown in Figure 1.

TABLE I. DGS OUTPUT RATIO AND CAPACITIES

\begin{tabular}{|c|c|c|c|c|}
\hline number & 1 & 2 & 3 & 4 \\
\hline $\begin{array}{c}\text { DG } \\
\text { capacity } \\
(\mathrm{MW})\end{array}$ & 742 & 1486 & 2230 & 2970 \\
\hline Ratio(\%) & 20 & 40 & 60 & 80 \\
\hline
\end{tabular}

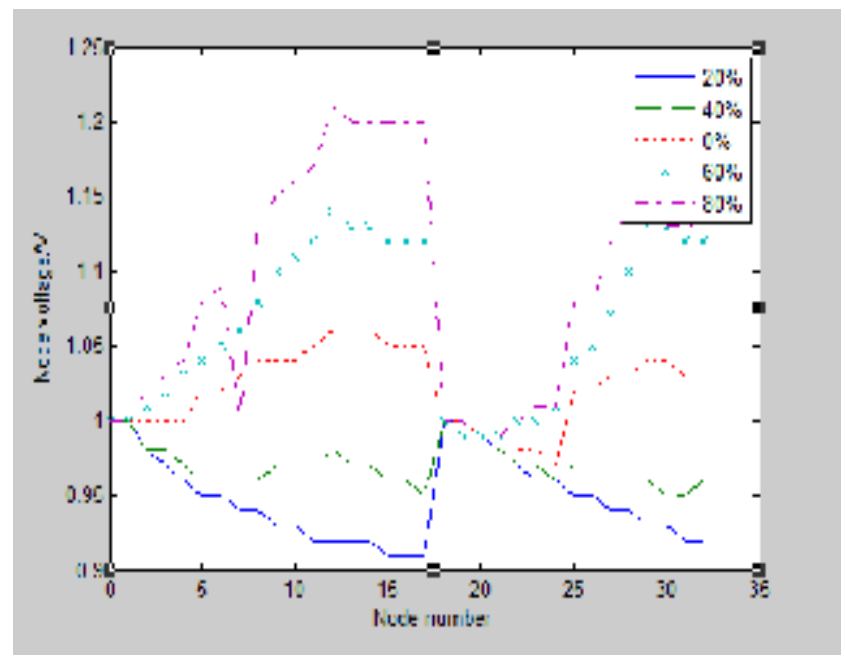

Figure 1. Simulation results of different DG capacity and ratio

This simulation result shows that the effect caused by the DG capacity change is obvious in the distribution network system. After the access of distributed power supply, the voltage on the load node increased. Because of the reduced power transmission in the power feeders and the reactive power supply provided by the DG, the penetration of DG is beneficial to the load node voltage. When the DG output at a certain range, a higher total output can give the load node voltage a greater support. If the DG power output exceed the limits, the level of the node voltage may beyond the permissible value. As shown in figure 3 when the ratio across $60 \%$, the node voltages are severely over voltage limit[7]. But as the DG capacity go on increasing, the node voltage will reduce. The power flow will change as the connection of DGs 
into the distribution network system. In order to control this change, the capacity of the distributed generators should be limited. In this paper only considering voltage stability requirements, DG access capacity should be around $40 \%$ of the total system capacity.

\section{B. Impact on voltage in different DG positions}

Maintain the output of each DG at a constant level and only to change the position in the distribution network system. According to the analysis above take the DG power output level at a ratio of $40 \%$. Then change the DG position to study the distribution network voltage. For better understanding, only take one DG into consideration. Table 2 presents the different DG positions and the simulation result is shown in figure 2 .

TABLE II. DIFFERENT DG POSITIONS

\begin{tabular}{|c|c|c|c|c|}
\hline Number & 1 & 2 & 3 & 4 \\
\hline $\begin{array}{c}\text { DG } \\
\text { position }\end{array}$ & 2 & 8 & 12 & 31 \\
\hline
\end{tabular}

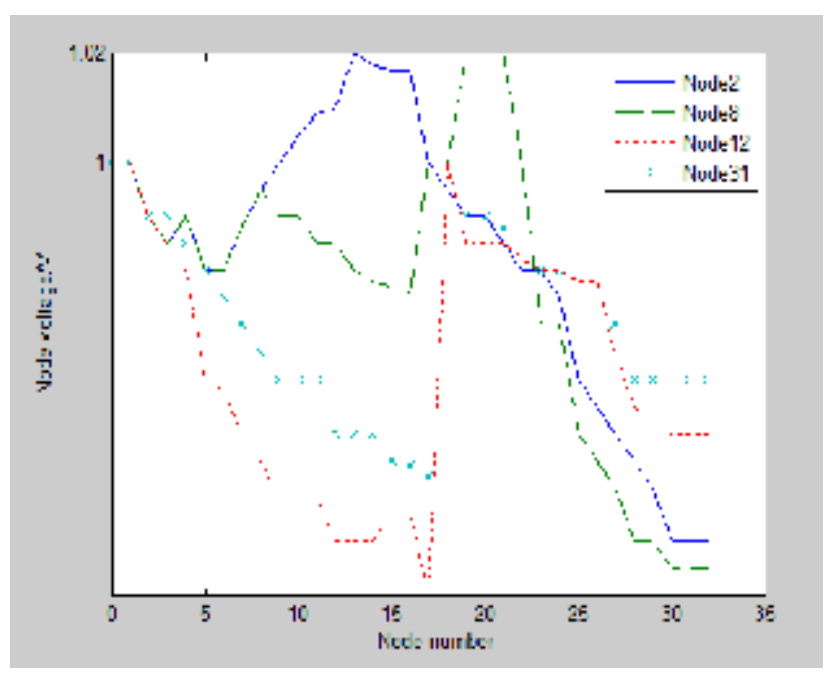

Figure 2. Simulation result of different DG positions

According to the simulation result, the different DG positions have different effects to the node voltage. The closer DG to the bus, the smaller effects will be caused. And the voltage will raised if the DG injected the distant node. This is likely to exceed the rated voltage limit and cause many adverse effects to the users. When the DG quit running, it also will cause the node voltage increase at the end of the feeder. Therefore it is inappropriate to inject DG at the end of the feeder and it is reasonable to place the DG in the middle of the feeder[8]. In this simulation the best injecting node is 28 .

\section{Impact on voltage in different power factor}

Maintain the DG capacity and position unchanged and take different power factors into consideration. Table 3 presents the different power factors and the simulation result is shown in Figure 3.
TABLE III. DIFFERENT POWER FACTORS

\begin{tabular}{|l|c|c|c|c|}
\hline number & 1 & 2 & 3 & 4 \\
\hline $\begin{array}{l}\text { DG } \\
\text { power } \\
\text { factor }\end{array}$ & 0.7 & 0.85 & 0.85 & 0.7 \\
(lagging) & (lagging) & (leading) & (leading) \\
\hline
\end{tabular}

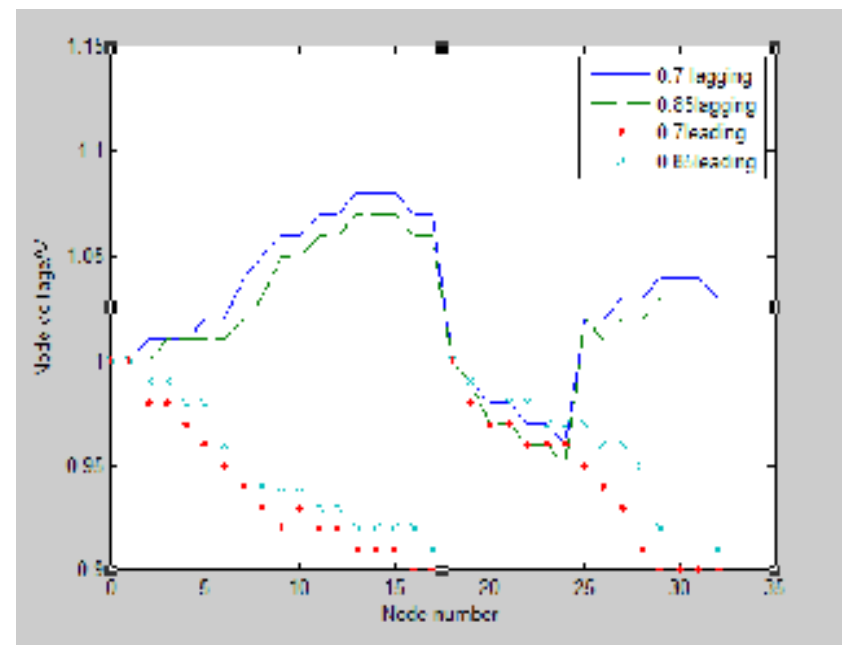

Figure 3. Simulation result of different power factors

According to the simulation result the voltage quality can be affected by the DG connected to the distribution network system. As shown in figure3, lagging[9] power factor can better adjust the voltage level than leading power factor. When DG with a leading power factor join up the distribution network system, the node voltage will not change too much and DG has little effect in improving the voltage level. In some extreme situation the voltage may reduce. If the DG has a lagging power factor, it can compensate the reactive power, thus the current transmitted in the feeder lines can reduce. As a result the node voltage level will increase.

The change process of the power factor is also a process that DG absorbs reactive power to export reactive power. When the reactive power absorbed by the DGs is more than the reactive power absorbed by the load, the maximal voltage will appear where the DG injected. The greater output of reactive power the higher voltage level the node will get. In practice, a regulator device will be added into the distribution network system to prevent the over-limit[10] voltage.

\section{CONCLUSION}

In this paper, the impacts caused by the DG are verified comprehensively through the MATLAB Simulation[11]. A certain capacity of distributed generation connected to the distribution network can cause a significant effect on the feeder. The degree caused by the DG is related to the total capacity of distributed generation, position of DG and the DG power factor. The traditional distribution network is deployed radically and the feeder voltage decreases along with the trend direction. With the DG inserted, the reduction of transmission power feeder and reactive power of DG may cause the load node voltage to increase under steady state condition.

DG location and design of a distributed system[12] capacity is an indispensable prerequisite. For the 
distributed power generally inserted into the user side, DG capacity the users needed and the circumstance near the users must be considered and then decide the generation mode. When decided locations of the distributed generations, not only the surrounding energy, transportation, geographical and environmental factors, but also the rationality of the DG location shall be considered. If we can control the voltage in the distribution network at a suitable level, the economical efficiency, reliability and flexibility of the distribution system can be improved significantly.

\section{REFERENCES}

[1] A. Jha, R. Gharpurey, and P. Kinget, "Quadrature-DAC based pulse generation for UWB pulse radio transceivers," WIT press,Jan. pp201-210.

[2] Sanghoon Sim, Dong-Wook Kim, and Songcheol Hong, "A CMOS UWB Pulse Generator", Proc. IEEE Symp. Electric Science, Nov. 2010, pp.234-258.

[3] C. Chang, S. Jung, S. Tjuatja, J. Gao, and Y. Joo, in: 49th IEEE Int. Midwest Symp.
[4] Hillmon Peter Ladner Garcia, "Photovoltaic based on distributed generation as a demand response strategy in puerto rico", CA: Energy Science,2008.

[5] B. Dupont, K. Dietrich, C. De Jonghe, "Impact of residential demand response on power system operation," CA: WCEE2013.

[6] Christopher J. Bennett, Rodney A. Stewart, Jun Wei Lu, "Distributed generator and resource," Energy, vol.5, pp. 574-601, 2007.

[7] Prasert Sinsukprasert, "Effects of demand side management on power system reliability," Science, vol.78, pp. 109-120,2011.

[8] Fernando Olsina, Rolando Pringles, Carlos Larisson, "A strategy on the distribution network about power output," Energy Policy, pp. 12-16, 2006.

[9] Mario L. Ferrari, Alberto Traverso, Matteo Pascenti, "Impacts of the access of distribution generator," Energy Conversion and Management, vol.12, pp.19-22, 2014.

[10] M.A. Abdullah, A.P. Agalgaonkar, K.M. Muttaqi, in: Applied Energy(2014)

[11] Keivan Navi, Mehrdad Maeen, Vahid Foroutan, "Efficiency of the distributed resource," CA:VLSI,2009.

[12] Tonko Garma, Silvestar Šesnić, "Electrical Power and Energy Systems," IEEE Press, Oct. 2009, pp.77-86. 